\section{Neuronal Potassium Channel Openers Flupirtine (SNEPCO) in the Treatment of the Alcohol Withdrawal without Psychiat- ric Comorbidity Randomized, Double-Blind, Placebo-Con- trolled Study}

\author{
Nadir A Aliyev $^{1 *}$ and Zafar Aliyev ${ }^{2}$ \\ ${ }^{1}$ Department of Psychiatry and Addiction, Azerbaijan State Advanced \\ Training Institute for Doctors, Baku, Azerbaijan
}

${ }^{2}$ Department of Psychiatry, Azerbaijan Medical University, Baku, Azerbaijan

\begin{abstract}
Objective

Alcohol withdrawal is highly prevalent in population. However, the effect of flupirtine on patients with alcohol withdrawal without psychiatric comorbidity has not been studied.

\section{Method}

Hundred patients (all men) were washed out from all medications. Each patient was randomized either to receive flupirtine (50 patients) for 2 weeks or matched on placebo (50 patients) in a double-blind manner. Eligible participants, in addition to meeting the criteria for alcohol withdrawal from Diagnostic and Statistical Manual of Mental Disorders, 5th Edition (DSM-5), were required to be between 18 and 50 years. Response was defined Alcohol Withdrawal Scale (AWS). Response effects with flupirtine and placebo were compared by using analysis of variance and $\mathrm{x} 2$ tests. Ten patients did not return for at least 1 subsequent assessment, and 90 patients dropped out (45 taking flupirtine and 45 taking placebo) in the valuables study group.
\end{abstract}

*Corresponding author: Nadir A Aliyev, Department of Psychiatry and Addic tion, Azerbaijan State Advanced Training Institute for Doctors, Baku, Azerbaijan, Tel: +994 124314033; E-mail: aliyevnadir@yahoo.com

Citation: Aliyev NA, Aliyev Z (2017) Neuronal Potassium Channel Openers Flupirtine (SNEPCO) in the Treatment of the Alcohol Withdrawal without Psychiatric Comorbidity Randomized, Double-Blind, Placebo-Controlled Study. J Addict Addictv Disord 4: 013.

Received: September 20, 2017; Accepted: November 20, 2017; Published: December 04, 2017

Copyright: @ 2017 Aliyev NA and Aliyev Z, et al., This is an open-access article distributed under the terms of the Creative Commons Attribution License, which permits unrestricted use, distribution, and reproduction in any medium, provided the original author and source are credited.

\begin{abstract}
Results
Of the 45 flupirtine-treated participants, all responded by 2 weeks versus 6 of the 45 placebo-treated participants $(P>0.001)$. The most common and problematic adverse effect in the flupirtine group was not. The results of our study showed high efficiency flupirtine in the treatment of alcohol withdrawal syndrome. The biochemical mechanism of action of flupirtine is similar to that of ritagabiline (chlorzoxazone).

Conclusion: The authors believe this to be the first double-blind placebo-controlled randomization study to test the efficacy of flupirtine in the management of alcohol withdrawal. These need to be replicated in a larger study group.
\end{abstract}

Keywords: Alcohol withdrawal; Flupirtine; Treatment

\section{Introduction}

According DSM-5 prevalence of the alcohol withdrawal approximately $50 \%$ of middle-class, highly functional individuals with alcohol use disorder have ever experienced a full alcohol withdrawal syndrome. Among individuals with alcohol use disorder who are hospitalized or homeless, the rate of alcohol withdrawal may be greater than $80 \%$. Fewer than $10 \%$ of individuals who develop alcohol withdrawal will ever develop dramatic symptoms (e.g., severe autonomic hyperactivity, tremors, alcohol withdrawal delirium). Tonic-clonic seizures occur in fewer than 3\% of individuals. It is known that symptoms of withdrawal may serve to perpetuate drinking behaviors and contribute to relapse, resulting in persistently impaired social and occupational functioning. The symptoms required urgently medically intervention. Alcohol withdrawal is associated with greater functional, social impairment and poor prognosis. The basis for the use of Flupirtine in the treatment of alcohol withdrawal syndrome was literature data suggesting that this drug reduce heavy alcohol drinking Retigabine a drug with the same mechanism of action is also tested in this area [1-5].

The aim of our study was to evaluate the effect of flupirtine in alcohol withdrawal is highly prevalent in population. However, possibility of using flupirtine (cadadolone) - SNEPCO (Selective Neuronal Potassium Channel Opener) - in the therapy alcohol withdrawal was not study. The work was carried away with in a double-blind placebo-controlled design. This is a report on a randomized, double-blind, placebo-controlled trial of neuronal potassium channel openers flupirtine (cadadolone).

\section{Materials and Methods of Research}

In accordance with the Helsinki Declaration of the World Medical Association "Recommendations for doctors engaged in biomedical research involving people", adopted by the $18^{\text {th }}$ World Medical Assembly (Finland, 1964, revised in Japan in 1975, Italy - 1983, Hong Kong - 1989, the South African Republic - 1996, Edinburgh - 2000); The Constitution of the Republic of Azerbaijan, the Law "On Psychiatric Assistance" (adopted on 12.06.2001, with amendments and additions - 11.11.2011, Decisions of the Cabinet of Ministers of the Republic of Azerbaijan No. 83, dated April 30, 2010 "On Approval of the Rules for Conducting Scientific, Preclinical and Clinical studies of medicines" are established: 
- The conditions of the conducted researches corresponded to the generally accepted norms of morality, the requirements of ethical and legal norms, as well as the rights, interests and personal dignity of the participants of the studies were observed

- Conducted research is adequate to the topic of research work

- There is no risk for the subject of research

- Participants in the study were informed about the goals, methods, expected benefits of the study and associated with risk and inconvenience in the study

- The subject's informed consent about participation in the research was received

The decision of the Ethical Committee at the Azerbaijan Psychiatric Association on the article of NA. Aliev, Z.N. Aliev "Selective Neuronal Potassium Channel Opener flupirtine (SNEPCO) in treatment-resistant epilepsy in adults" submitted for publication in psychiatric journals: in connection with compliance with its legislative requirements and regulatory documents is to approve the article by N.A. Aliyev, Z.N. Aliev "Selective Neuronal Potassium Channel Opener flupirtine (SNEPCO) in treatment of alcohol withdrawal". We examined 100 patients with (FI0.239). Patients were observed at the Mental Health Center of the Ministry of Health of the Republic of Azerbaijan.

According DSN-5 [6] the essential feature of alcohol withdrawal is the presence of a characteristic withdrawal syndrome that develops within several hours to a few days after the cessation of (or reduction in) heavy and prolonged alcohol use (Criteria A and B). The withdrawal syndrome includes two or more of the symptoms reflecting autonomic hyperactivity and anxiety listed in Criterion B, along with gastrointestinal symptoms. Withdrawal symptoms cause clinically significant distress or impairment in social, occupational, or other important areas of functioning (Criterion C). The symptoms must not be attributable to another medical condition and are not better explained by another mental disorder (e.g., generalized anxiety disorder), including intoxication or withdrawal from another substance (e.g., sedative, hypnotic, or anxiolytic withdrawal) (Criterion D). Symptoms can be relieved by administering alcohol or benzodiazepines (e.g., diazepam). The withdrawal symptoms typically begin when blood concentrations of alcohol decline sharply (i.e., within 4-12 hours) after alcohol use has been stopped or reduced. Reflecting the relatively fast metabolism of alcohol, symptoms of alcohol withdrawal usually peak in intensity during the second day of abstinence and are likely to improve markedly by the fourth or fifth day.

It is known that (CIWA-Ar) score divided to three stages:

- $<8$ Mild withdrawal;

- $<$ 8-25 Moderate to severe withdrawal and

- 25 Very severe withdrawals [Drug \& Alcohol Services Council 2001: Revised 2003] our patients correspond to the moderate to severe alcohol withdrawal

Hundred patients (all men) were washed out from all medications. Each patient was randomized either to receive flupirtine (50 patients) for 2 weeks or matched on placebo (50 patients) in a doubleblind manner. Eligible participants were required to be between 18 and 65 years. Ten patients did not return for at least 1 subsequent assessment, leaving 90 patients (45 taking flupirtine and 45 taking placebo) in the valuables study group. There were premature dropouts, in each group 5 because of noncompliance with the visits. Flupirtine and placebo were dispensed in identical appearing capsules; patients randomized to the placebo group took the same number of capsules as those assigned to the flupirtine group.

In our studies we used Flupirtine forte (flupirtinum) was prescribed the first 7 days of $200 \mathrm{mg} 3$ times a day, then $400 \mathrm{mg} 3$ times in day, in capsules for 14 days (Produced by Pliva Krakow, Pharmaceutical Plant AO). This dose was maintained until the end of the trial at week 2 . Starting on week 0 , patients received study medication. Capsules were supplied in numbered bottles containing study medication as determined by a random number sequence. The randomization list was held by the senior investigator outside the treatment team. No communication regarding the status of patients under study was permitted the unblended investigator and the other investigators, save that the unblended investigator was informed if patients complained of any adverse effects from the study medication. Hospital staff with no clinical responsibilities and knowledge of the patients oversaw the assignment procedure and assigned medication in sequential order, strictly following the randomized list. The treating psychiatrist did not have access to the list. Both the patient and the treating psychiatrist were not aware of the antipsychotic being prescribed. Patients were excluded if they displayed an acute systemic medical disorder or a medical disorder requiring frequent changes in medication and if they displayed a history of seizures, cardio-vascular disease, structural brain damage from trauma, focal neurological signs on examination, or evidence of any progressive neurological disorder, and substance dependence (except tobacco). Contraindications in our patients were following: patients with history of hypersensitivity to flupirtine, hepatic encephalopathy, cholestasis, myasthenia gravis, chronic alcoholism, primary biliary cirrhosis, and liver disease. Patients underwent physical examination, electrocardiography (if $>40$ years), and laboratory analyses, including hematological measures, partial thromboplastin time, and urinalysis. All patients were evaluated on weeks 0 and 2 weeks of the study by different psychiatrists. The adverse effects were recorded by spontaneous reports. Patients attended 4 visits: initial screening (randomization (week 0)), and 2 times further visits at weeks 2. Data for clinical assessments were collected at weeks 0 and 2. Data for adverse events were spontaneous complaints at each visit. Patients were also requested to report immediately at any onset of possible rash or other skin reactions.

The patients did not receive any antidepressant or anticonvulsant drugs for a period of 2 weeks before the study. All of the patients provided with the written informed consent after a full explanation of the protocol design that had been approved by the local ethics committee (Azerbaijan Psychiatric Association approved this study). Patients who were not able to give reliable informed consent were excluded from the study. Patients were recruited from January 2016, and follow up was completed by January 2017. Dropout rates and reasons for dropping out were also recorded.

Comparison between the groups at baseline was performed using the Mann-Whitney test. Analysis of response refers to the last observation carried forward for all patients who had valuables efficacy at baseline and with treatment. The responder analysis was conducted by using the $\mathrm{x} 2$ and Analysis of Variance (ANOVA) according to Glantz [7]. 


\section{Results}

Characteristics of the patients randomly assigned to the 2 treatment groups are also shown in table 1.

\begin{tabular}{|c|c|c|c|c|c|}
\hline \multirow{3}{*}{$\begin{array}{c}\text { Indicators } \\
\text { Age, mean (SD), y }\end{array}$} & \multicolumn{4}{|c|}{ Treatment Groups } & \multirow{3}{*}{$\begin{array}{c}\text { Statistics P } \\
\text { NS } \\
\text { NS }\end{array}$} \\
\hline & \multicolumn{2}{|c|}{ Flupirtine Group $\mathbf{n}=\mathbf{5 0}$} & \multicolumn{2}{|c|}{ Placebo Group n=50 } & \\
\hline & 38.0 & $(00.2)$ & 37.4 & $(9.8)$ & \\
\hline Duration of illness, & 16.5 & $(13.0)$ & 18.5 & $(12.2)$ & NS \\
\hline \multicolumn{6}{|l|}{ Mean (SD), y } \\
\hline Age at onset, & 15.0 & (7.0) & 15.5 & (7.6) & NS \\
\hline \multicolumn{6}{|l|}{ Mean (SD), y } \\
\hline \multicolumn{6}{|l|}{ Education, n (\%) } \\
\hline Primary school & 28 & (70) & 30 & (75) & NS \\
\hline Secondary school & 12 & (30) & 10 & (25) & NS \\
\hline \multicolumn{6}{|l|}{$\begin{array}{c}\text { Marital status, } \\
\text { n (\%) }\end{array}$} \\
\hline Never married & 10 & (25) & 10 & (25) & NS \\
\hline Married & 20 & (50) & 18 & (45) & NS \\
\hline $\begin{array}{l}\text { Divorced or } \\
\text { separated }\end{array}$ & 10 & (25) & 12 & (30) & NS \\
\hline \multicolumn{6}{|l|}{$\begin{array}{c}\text { Employment status, } \\
\mathrm{n}(\%)\end{array}$} \\
\hline Unemployed & 40 & (100) & 40 & (100) & NS \\
\hline
\end{tabular}

Table 1: Demographic and Clinical Characteristics of the Study Sample $(\mathrm{N}=100)$.

Indicators Treatment groups Statistics P.

As shown in table 1, in the baseline Mann-Whitney $\mathrm{U}$ test, there were no significant differences between 2 groups. Statistical differences between the 2 groups are not significant.

Hundred patients (all men) completed the study and were included in the analyses of efficacy (Table 2). As shown in table 2, patients did not return for at least 1 subsequent assessment, leaving 90 patients (45 taking Flupirtine and 45 taking placebo) in the valuables study group. There were 5 premature dropouts, in the first group and 5 in the second (placebo) group. In both groups on 5 because of noncompliance with the visits. Hence, 45 in the first group and 45 in the second group completed the analysis. Mean total scores on the CIWA-Ar scale during treatment of alcohol withdrawal are shown in table 2. Mean total scores on the CIWA-Ar for 2 weeks were significantly different between the 2 groups. At the end of 2 weeks of therapy significantly decreased the rating in the CIWA-Ar.

\begin{tabular}{|c|c|c|c|}
\hline \multirow{2}{*}{ Intention to Treat } & \multicolumn{2}{|c|}{ Treatment Groups } & Statistics P \\
\cline { 2 - 4 } & $\begin{array}{c}\text { Flupirtine } \\
\text { Group } \mathbf{n}=\mathbf{5 0}\end{array}$ & $\begin{array}{c}\text { Placebo Group } \\
\mathbf{n = 5 0}\end{array}$ & NS \\
\hline Completers analysis means $=90$ & 45 & 45 & NS \\
\hline $\begin{array}{c}\text { (CIWA-Ar) score at start of the } \\
\text { study (wk 0) }\end{array}$ & $17.5 \pm 1.5$ & $17.5 \pm 1.5$ & NS \\
\hline $\begin{array}{c}\text { (CIWA-Ar) score at end of the } \\
\text { study (wk 2) }\end{array}$ & $2.0 \pm 0.1$ & $20.0 \pm 1.7$ & $\mathrm{P}>0.001$ \\
\hline
\end{tabular}

Table 2: Mean CIWA-Ar score scale during treatment.

Mann-Whitney U test, significant difference between the flupirtine and the placebo groups Results of the Treatment (Observed and expected number from W2 analysis) shown in the table 3.

\begin{tabular}{|c|c|c|c|}
\hline \multicolumn{1}{|c|}{} \\
\hline Treatment Groups & Improvement & No Improvement & Total \\
\hline Flupirtine & $35(15.60)$ & $10(20.43)$ & 45 \\
\hline Placebo group & $6(16.43)$ & $39(22.68)$ & 45 \\
\hline Total & 41 & 49 & 90 \\
\hline
\end{tabular}

Table 3: Results of the Treatment (Observed and expected number from the W2 Analysis).

Expected numbers indication in the brackets, $\mathrm{W} 2=22.68, \mathrm{df}=1$. $\mathrm{P}<0.001$

Adverse effects reported by patients who received Flupirtine and placebo. Adverse Effect Flupirtine Group $(\mathrm{n}=45)$, n (\%) Placebo Group $(\mathrm{n}=45), \mathrm{n}(\%) \mathrm{P}^{*}$

\begin{tabular}{|c|c|c|c|}
\hline Adverse Effect & $\begin{array}{c}\text { Flupirtine Group } \\
(\mathbf{n}=\mathbf{4 5}) \mathbf{n}(\mathbf{\%})\end{array}$ & $\begin{array}{c}\text { Placebo Group (n } \\
\mathbf{=} \mathbf{4 5}) \mathbf{n}(\mathbf{\%})\end{array}$ & $\mathbf{P}^{*}$ \\
\hline Dizziness & $6(14)$ & $35(78)$ & $>0.001$ \\
\hline Headache & $2(4)$ & $16(35)$ & $>0.001$ \\
\hline Fatigue & $3(7)$ & $40(89)$ & $>0.001$ \\
\hline Perspiration & $5(11)$ & $35(78)$ & $>0.001$ \\
\hline Sedation & $5(11)$ & $3(7)$ & NS \\
\hline Nausea & $1(2)$ & $20(44)$ & $>0.001$ \\
\hline Blurred vision & $4(9)$ & $14(31)$ & $>0.001$ \\
\hline Sleepiness & $1(2)$ & $38(84)$ & $>0.001$ \\
\hline Vomiting & $8(18)$ & $25(55)$ & $>0.001$ \\
\hline
\end{tabular}

Table 4: Adverse effects reported by patients who received Flupirtine and placebo.

Analysis of statistical significance was carried out with criteria of the alternative changes.

NS - not significant

Table 3 shows the results of the treatment on the W2 analysis. As shown in table 3, a statistically significant difference in improvement was observed in the first group (Flupirtine) compared with that in the second group (placebo). It is necessary to indicate that, in patients from the first group, compared with those in the second group, statistically significant adverse effects were observed (Table 4). Headache in the first group of patients was significantly lower than that in the second group of patients.

The data obtained by us in connection with a small number of patients and a short period of observation ( $2 \mathrm{wk}$ ) should be considered preliminary. The next stage of the work will be carried out on a large number of patients and longer duration of observation, with a placebo-controlled, doubleblind method.

\section{Discussion}

Flupirtine was administered as an alternative analgesic for opioids and NSAIDs. Subsequently, several other actions have been identified, such as muscle relaxation and neuroprotective activity. Flupirtine acts indirectly as an N-Methyl-D-Aspartate (NMDA) receptor antagonist by activating $\mathrm{K}^{+}$channels [8].

Flupirtine causes a dose-dependent decrease in NMDA-induced glutamate induced by an increase in the intracellular $\mathrm{Ca}^{++}$concentration [9]. It binds and activates the G-protein bound to the $\mathrm{K}^{+}$channels directed inwards. Activation of this channel leads to hyperpolarization of the neuronal membrane, and the neuron becomes less excitable; thus, stabilization of the resting neuron membrane is observed [10]. Drugs that activate this channel are called Selective Neuronal 
Potassium Channel Openers (SNEPCO), and flupirtine is a prototype [8]. Experimental evidence suggests that flupirtine can inhibit channel opening by acting as an oxidant in the redox site of the NMDA receptor [11]. This action inhibits the transfer of nociceptive impulses when neurons are excited. The central effect of flupirtine is based on 4 main effects [12].

\section{Analgesic action}

Flupirtine was introduced as an alternative analgesic to opioids and NSAIDs. Subsequently, multiple other actions such as muscle relaxation and neuroprotective activity were identified. Flupirtine acts indirectly as N-Methyl-D-Aspartate (NMDA) receptor antagonist by activation of $\mathrm{K}^{+}$channels [13]. Flupirtine causes a dose-dependent reduction of NMDA receptor mediated glutamate induced rise in intracellular $\mathrm{Ca}^{++}$concentration [9]. It binds to and activates G- protein coupled inwardly rectifying $\mathrm{K}^{+}$channels. Activation of this channel leads to hyperpolarization of neuronal membrane and the neuron becomes less excitable; thus, there is stabilization of resting neuronal membrane [14]. The drugs activating this channel are called as Selective Neuronal Potassium Channel Openers (SNEPCO) and flupirtine is the prototype [8]. Experimental evidence suggests that flupirtine might suppress channel opening by acting as an oxidizing agent at the redox site of the NMDA receptor [11]. This action inhibits the transmission of nociceptive impulses during neuronal excitation.

\section{Muscle relaxant action}

The muscle relaxation is due to inhibition of both mono- and polysynaptic reflexes. The spinal polysynaptic flexor reflex, mediated by NMDA receptors, was depressed by flupirtine, whereas the monosynaptic Hoffmann reflex (H-reflex), mediated by non-NMDA receptors, was not influenced. Healthy human subjects responded with a significant reduction of both the early phase of the electrically elicited polysynaptic flexor reflex of pretibial muscles and the medium latency response of the toe-up paradigm after $2 \mathrm{~h}$ of $200 \mathrm{mg}$ of flupirtine. Flupirtine possesses analgesic as well as muscle-relaxing effect in same dose ranges; thus, it can be used in the treatment of painful diseases of the motor system presenting with spasticity and chronic musculoskeletal pain [15].

\section{Neuroprotective action}

Apoptosis, a programmed cell death, is caused by increased intracellular $\mathrm{Ca}^{++}$levels, mitochondrial dysfunction, cell membrane disruption, and finally nucleolysis. In vitro studies with primary cortical neurons from rat embryos have shown that lead acetate; prions like PrPsc, HIV coat protein gp120, and $\beta$ amyloid peptide will cause apoptotic cell death. But if preincubated with flupirtine, it completely protects apoptotic cell death caused by above agents in the neurons [16]. It has been found that flupirtine also antagonizes both glutamate and NMDA induced increase in intracellular levels of $\mathrm{Ca}^{++}$, as observed in vitro cultures of cortical and hippocampal neurons $[16,17]$. The expression of Bcl-2, an antiapoptotic agent, and glutathione, a scavenger of reactive oxygen, are reduced during glutamate or NMDA-induced apoptosis in cells. Flupirtine is found to increase the levels of Bcl-2 and glutathione in glutamate or NMDA-induced apoptosis of human Ntera/D1 (hNT) neurons as well as cultured retinal pigment cells [11]. Flupirtine reduced the expression of oncogenes and formation of reactive oxygen radicals in experimental models which explains its action of preventing ischemia induced apoptosis. This explains the role of flupirtine in future for treatment of neuroinfections such as Immune Deficiency Syndrome (AIDS), prion diseases, and neurodegenerative disease such as Alzheimer's [8].

\section{Antiparkinsonian action}

Flupirtine has an NMDA receptor antagonistic action and hence it was studied for its Antiparkinsonian effect as an adjuvant to L-3, 4-Dihydroxyphenylalanine (L-DOPA). Akinesia and muscular rigidity were produced in rats by giving reserving and $\alpha$ methyl p-tyrosine. Flupirtine was given alone and in combination with L-DOPA, it strongly reduced muscle rigidity and increased the ability of L-DOPA to reverse Akinesia [18]. In haloperidol-induced catalepsy, which is considered as a model of Parkinson's disease, flupirtine alone and in combination with L-DOPA exerted a potent and cataleptic effect $[14,17]$. However, human studies are not available till date to support this evidence. If studies are done to prove effectiveness in Parkinson's disease, it can be combined with L-DOPA.

\section{Special group}

Safety Flupirtina in pregnant women, lactating women and children less than 6 years is not established. If indicated in lactating women, breastfeeding should be discontinued. Flupirtine dose should be reduced to $50 \%$ in elderly patients and patients with renal and hepatic insufficiency [18].

\section{Contraindications}

Flupirtine avoids patients with hypersensitivity to flupirtine, hepatic encephalopathy, cholestasis, myasthenia gravis, chronic alcoholism, primary biliary cirrhosis and liver diseases.

\section{Benefits}

Musculoskeletal pain: Flupirtine was compared with placebo and standard analgesics to determine the efficacy and tolerability of analgesics. Post-marketing surveillance for flupirtine $200-300 \mathrm{mg} /$ day for 1 week, as assessed by visual analog pain scale. It was noted that the response rates were $94 \%, 89.4 \%$ and $85.9 \%$ for patients with acute, sub acute and chronic pain, respectively $[18,19]$.

Headache: Patients who had an inadequate response to conventional analgesics for chronic headache showed a better response to flupirtine [19]. The results in our study indicate that Flupirtine is more effective in treating alcohol withdrawal than placebo. Analysis of statistical significance was carried out with criteria of the alternative changes. Indicates significant this has led us to investigate other potential pharmacotherapies, in particular the anticonvulsant Flupirtine [20]. Flupirtine acts at the presynaptic membrane to reduce the release of glutamate, and it has been shown to reverse depersonalization-related phenomena induced by the NMDA receptor antagonist ketamine in a healthy individual. The mechanisms of Flupirtine may be connected with the influence of neuronal activity and glutamate and GABA transmission.

In one hand Flupirtine mechanism of action similar; as a retigabine, and in other hand, given that retigabine is an FDA-approved drug, its use in a clinical trial on alcohol use disorder is theoretically feasible [3]. Because retigabine is also tested in this area. Thus our date correlated with some literature date [1-3, 21-24]. These date that indicated that the potassium channel may be as targets for therapeutic intervention and novel therapeutic strategies for alcohol and drug addiction retigabine is also tested in this area. We suggest that the Flupirtine mechanism of action on alcohol is same as a retigabine. 
The date of Lavreskaya EF [25] shown that an increase in the membrane conductivity for $\mathrm{K}^{+}$ions (i.e., activation-opening of the potassium channels) causes neuronal hyperpolarization and, in most cases, reduces the frequency of neuronal excitation, exerting a strong inhibitory effect on the excitability of neurons. Potassium channels control the membrane potential of rest and, therefore, play an important role in regulating the excitability of neurons. These data served the basis for the present study. She wrote: "It is necessary to emphasize several important points relating to those functions in which a special role belongs to the potassium channels

1. The main function of potassium channels is the catalysis of the transfer of potassium ions through the membrane, which ensures the existence of a difference in electrical potentials between the inner and outer sides of the membrane in all living cells. The fulfillment of this functional role is ensured by the high conductivity and high selectivity of these channels.

2. In addition to this potential-forming role, potassium channels participate in the process of depolarization of the membrane of electro excitable cells, accelerating repolarization and as if returning the potential to a resting level. In rare cases, membrane depolarization can occur due to a decrease in potassium permeability.

3. All the mentioned above determines the special role played by the change in the potassium permeability of the membrane providing many physiological processes, in particular, excitation in nerve and muscle cells, the creation of rhythms in neuronal and cardiac pacemakers, the secretion of hormones by glandular cells and mediators by nerve endings, certain types of reception (For example, light), memory processes and many others, not fully clarified yet.

4. The multiplicity of the channels regulating the potassium conductivity of the membrane indicates a special plasticity of this membrane function. The variety of potassium channels is reflected in various sensitivity to pharmacological agents, blockers. This opens important prospects for the selective impact on various components of potassium conductivity and, thus, on the processes in which they participate".

In such a way the available up-to-date literature data indicate an increase in the membrane conductivity for $\mathrm{K}^{+}$ions (i.e., activation-opening of the potassium channels) cause neuronal hyperpolarization and, in most cases, reduces the frequency of neuronal excitation, exerting a strong inhibitory effect on the excitability of neurons. Potassium channels control the membrane potential of rest and, therefore, play an important role in regulating the excitability of neurons. Four limitations should be noted. First was our small study group and we recommending that these results be replicated in a larger group so that effect sizes can be more precisely estimated. Second, it is necessary to conduct this study for the possible generalizability of these data on a sample of women with alcohol withdrawal. Third, the absence of systemic side effects indeed to future investigations. Fourth, it is necessarily study, side effects of the CNS. The fifth, further research is needed to establish the optimal dosage. Notwithstanding these limitations, this study suggests that Flupirtine is efficacious and well tolerated in the treatment of alcohol withdrawal. In any event, pending a further understanding of Flupirtine' s mechanisms of action, the present data suggest that this drug is a useful new agent for the treatment of alcohol withdrawal, in patients who have failed to respond to other traditional drugs. It will be important to explore further effects of Flupirtine in other disorders treated with Flupirtine.
However, our results show certain limited duration of the trial. Nevertheless, these findings provide evidence that Flupirtine treatment is well tolerated and may be proposed as an effective therapeutic strategy to improve outcome in treatment of alcohol withdrawal. Some authors established, that chlorzoxazone, reduce NAcb SK channel function with chronic ethanol intake enhances NAcb excitability and ethanol intake, and the FDA-approved SK activator chlorzoxazone reduces excessive ethanol intake $[24,26]$. Other authors indicated that small conductance calcium-activated potassium type 2 channels regulate alcohol-associated plasticity of glutamatergic synapses [25].

\section{Conclusion}

The results of our study showed high efficiency flupirtine in the treatment of alcohol withdrawal syndrome. The biochemical mechanism of action of flupirtine is similar to that of ritagabiline (chlorzoxazone). Despite the tremendous progress that has been made recently in elucidating the neurobiological basis of addiction, the expected subsequent therapeutic improvements have not been realized. Channel blockers represent a new goal that can be translated into research clinical studies in the near future, especially with the use of drugs already approved for other indications. The novelty of the work is the first application of the neuronal potassium channel openers in the treatment of alcohol withdrawal syndrome.

\section{Author Disclosure Information}

The authors declare that the article is submitted on behalf of all authors. None of the material in the article has been published previously in any form and none of the material is currently under consideration for publication elsewhere other than noted in the cover letter to the editor. Authors declare no financial and personal relationship with other people or organizations that could inappropriately influence this work. All authors contributed to and have approved the final article.

\section{The authors declare no conflicts of interest}

No sponsor provided funding for this study. Mental Health Center of the Ministry of Health of the Republic of Azerbaijan provided the outpatient unit, the material for clinical and neuropsychological assessments, and electronic resources.

\section{Acknowledgments}

The authors would like to thank staff of the Mental Health Center of the Ministry of Health of the Republic of Azerbaijan.

\section{References}

1. Rinker JA, Fulmer DB, Trantham-Davidson H, Smith ML, Williams RW, et al. (2017) Differential potassium channel gene regulation in $\mathrm{BXD}$ mice reveals novel targets for pharmacogenetic therapies to reduce heavy alcohol drinking. Alcohol 58: 33-45.

2. Addolorato G, Leggio L, Hopf FW, Diana M, Bonci A (2012) Novel therapeutic strategies for alcohol and drug addiction: focus on GABA, ion channels and transcranial magnetic stimulation. Neuropsychopharmacology 37: 163-177.

3. Knapp CM, O’Malley M, Datta S, Ciraulo DA (2014) The Kv7 potassium channel activator retigabine decreases alcohol consumption in rats. Am J Drug Alcohol Abuse 40: 244-250.

4. Zwierzyńska E, Andrzejczak D, Pietrzak B (2016) Does retigabine affect the development of alcohol dependence?--A pharmaco-EEG study. Neurosci Lett 611: 6-13. 
5. Hopf FW, Simms JA, Chang SJ, Seif T, Bartlett SE, et al. (2011) Chlorzoxazone, an SK-type potassium channel activator used in humans, reduces excessive alcohol intake in rats. Biol Psychiatry 69: 618-624.

6. American Psychiatric Association (2013) Diagnostic and Statistical Manual of Mental Disorders: Dsm-5. Amer Psychiatric Pub Incorporated, Arlington, USA.

7. Glantz AS (2011) Primer of Biostatistics (7thedn). McGraw Hill Professional, New York, USA. Pg no: 1-320.

8. Kornhuber J, Maler M, Wiltfang J, Bleich S, Degner D, et al. (1999) [Neuronal potassium channel opening with flupirtine]. Fortschr Neurol Psychiatr 67: 466-475

9. Rupalla K, Cao W, Krieglstein J (1995) Flupirtine protects neurons against excitotoxic or ischemic damage and inhibits the increase in cytosolic $\mathrm{Ca}^{2+}$ concentration. Eur J Pharmacol 294: 469-473.

10. Jakob R, Krieglstein J (1997) Influence of flupirtine on a G-protein coupled inwardly rectifying potassium current in hippocampal neurones. $\mathrm{Br}$ J Pharmacol 122: 1333-1338.

11. Osborne NN, Cazevieille C, Wood JP, Nash MS, Pergande G, et al. (1998) Flupirtine, a nonopioid centrally acting analgesic, acts as an NMDA antagonist. Gen Pharmacol 30: 255-263.

12. Harish S, Bhuvana K, Bengalorkar GM, Kumar TN (2012) Flupirtine: Clinical pharmacology. J Anaesthesiol Clin Pharmacol 28: 172-177.

13. Schmidt WJ, Schuster G, Wacker E, Pergande G (1997) Antiparkinsonian and other motor effects of flupirtine alone and in combination with dopaminergic drugs. Eur J Pharmacol 327: 1-9.

14. Schuster G, Schwarz M, Block F, Pergande G, Schmidt WJ (1998) Flupirtine: A review of its neuroprotective and behavioral properties. CNS Drug Rev 4: 149-164.

15. Zimmer G, Balakirev M, Hofmann M, Woodcock BG, Pergande G (1998) Evidence that the cytoprotective action of the triaminopyridine flupirtine involves increases in Ca2+ uptake and ATP synthesis in mitochondria. Br J Pharmacol 123: 1154-1158.
16. Schwarz M, Nolden-Koch M, Purr J, Pergande G, Block F (1996) Antiparkinsonian effect of flupirtine in monoamine-depleted rats. J Neural Transm (Vienna) 103: 581-590.

17. Devulder J (2010) Flupirtine in pain management: pharmacological properties and clinical use. CNS Drugs 24: 867-881.

18. Friedel HA, Fitton A (1993) Flupirtine: A review of its pharmacological properties, and therapeutic efficacy in pain states. Drugs 45: 548-569.

19. Mueller-Schwefe G (2003) [Flupirtine in acute and chronic pain associated with muscle tenseness. Results of a postmarket surveillance study]. Fortschr Med Orig 121: 11-18.

20. Aliyev NA, Qafarov TA, Aliyev ZN (2017) Neuronal potassium channel openers flupirtine (SNEPCO) in the treatment of the pharmacoresistant epilepsy. Abstracts of the 25th European Congress of Psychiatry. Supplement April 4: 674.

21. Gunthorpe MJ, Large CH, Sankar R (2012) The mechanism of action of retigabine (ezogabine), a first-in-class $\mathrm{K}+$ channel opener for the treatment of epilepsy. Epilepsia 53: 412-424.

22. Marty VN, Spigelman I (2012) Long-lasting alterations in membrane properties, $\mathrm{K}^{+}$currents, and glutamatergic synaptic currents of nucleus accumbens medium spiny neurons in a rat model of alcohol dependence. Front Neurosci 6: 86.

23. Blednov YA, Stoffel M, Chang SR, Harris RA (2001) Potassium channels as targets for ethanol: studies of G- protein-coupled inwardly rectifying potassium channel 2 (GIRK2) null mutant mice. J Pharmacol Exp Ther 298: 521-530.

24. Garcia ML, Kaczorowski GJ (2005) Potassium channels as targets for therapeutic intervention. Sci STKE 302: 46.

25. Lavretzky EF (1985) Pharmacological regulation of mental processes.

26. Mulholland PJ, Becker HC, Woodward JJ, Chandler LJ (2011) Small conductance calcium-activated potassium type 2 channels regulate alcohol-associated plasticity of glutamatergic synapses. Biol Psychiatry 69: 625-632. 